\title{
Screening of social phobia symptoms in a sample of Egyptian university students
}

\author{
Menan Abdel Maksoud Rabie ${ }^{1}$ \\ http://orcid.org/0000-0001-9318-7537 \\ EMAN SHORAB 1 \\ https://orcid.org/0000-0003-4845-7686 \\ DiNA ELGABRY1 \\ https://orcid.org/0000-0001-9783-6119 \\ KARIM ABdel AzIZ2 \\ https://orcid.org/0000-0002-7609-7756 \\ WalaA Mohamed SabrY ${ }^{1}$ \\ https://orcid.org/0000-0002-7361--5941 \\ Ola Aufa ${ }^{1}$ \\ hitps:///rrid.org/0000-0002-9048-404X \\ ReEm ElGhamry ${ }^{1}$ \\ https://orcid.org/0000-0003-4768-828X \\ GHADA HASSAN ${ }^{1}$ \\ https://orcid.org/0000-0002-1085-2050 \\ NAHLA NAGY 1 \\ https://orcid.org/0000-0003-3229-1001 \\ 1 Institute of Psychiatry, Faculty of Medicine, Ain Shams University, Cairo, Egypt. \\ 2 Department of Psychiatry, College of Medicine and Health Sciences, United Arab Emirates University, Al-Ain Emirates.
}

Received: 03/23/2018 Accepted: 04/02/2019

DOl: 10.1590/0101-60830000000188

\begin{abstract}
Background: Public speaking is a main requisite for academic achievement amongst university students. Epidemiological data on social phobia among University students in the Arab World are scarce. Objectives: To screen for social phobia symptoms in a representative sample of Egyptian university students, and to highlight some putative risk factors associated with them. Methods: Two thousand nine hundred and nineteen Egyptian university students participated in the study. They were screened for social phobia using Brief Social Phobia Scale (BSPS). Students total and subdomain scores on the BSPS were correlated with their demographic and scholastic variables. Results: Social phobia symptoms were estimated to be prevalent among (44\%) of the study sample. A significant $(p<0.05)$ negative correlation was found between students' age and avoidance scores on BSPS and between students' educational year and their scores on fear, avoidance and physiologic subdomains on the BSPS. Regression analysis pointed out to a number of predictive factors for the higher total BSPS scores such as female gender and being a medical student. Discussion: Our study demonstrated a high rate of social phobia symptoms in university students. It also demonstrated a number of significant predictive factors associated with the domains of social phobia such as gender, BMI, scholastic year and faculty type.
\end{abstract}

Rabie MAM et al. / Arch Clin Psychiatry. 2019;46(2):27-32

Keywords: Social phobia, social anxiety disorder, BSPS, university, students, Egypt.

\section{Introduction}

Social phobia consists of a persistent fear that individuals will be judged negatively or will behave in an embarrassing way in social situations ${ }^{1}$. It is the commonest type of anxiety disorders and is the third most common psychiatric disorder after major depression and alcohol dependence. Lifetime prevalence estimates for social phobia vary greatly worldwide and range from $0.4 \%$ to $20.4 \%$ in different studies. Rates of social phobia vary across studies due to various cultural \& social factors which are another important determinant of social anxiety disorder (SAD) especially in Arab and Islamic cultures ${ }^{2}$.

In the Arab Islamic world, social phobia is underestimated and underdiagnosed as social conformity and patriarchal and hierarchal orders dominate, where even small diversions from these rules are unacceptable, and individuals who do not conform are considered culturally deviant. Adherence to all social demands could be stressful and needs discipline and high self-control that is exercised at the expense of personal autonomy. The above-mentioned factors might increase the prevalence rate of SAD among Arabs and would make it more difficult to be discovered. In highly individualistic societies, like in the Western World, where one gains social advantage by asserting one's rights and leading the pack, even relatively low levels of social fear will be viewed as distressing and interfering. In collectivist societies like in the Arab World, where it is more socially advantageous to fit in with the community and express modesty, some degree of social anxiety would be viewed as positive and the disorder would only be defined at relatively high levels of social fear ${ }^{3}$.

Other factors that determine the rates of social phobia are; the diagnostic criteria used (DSM vs. ICD), variations in the method of assessment, rater experience in assessment ${ }^{2}$. Another factor is the number of phobic situations being evaluated with more situations evaluations leading to greater diagnostic sensitivity ${ }^{4}$.

Although it is a common psychiatric disorder, social phobia is often under-recognized and under-treated. It occurs more commonly in young people and these individuals are less likely to get married and more likely to get divorced. It is also associated with poorer educational achievements, unstable employment, higher work absenteeism and lower productivity ${ }^{5}$. 
Until recently, little research had examined the prevalence of social phobia in university students. Prevalence rates of social phobia in university students in different countries range from as low as $10 \%$ in the $\mathrm{UK}^{6}$ to as high as $36.8 \%$ in Nigeria ${ }^{7}$. Egypt is the most populated Arab country with a population of 91 million people (around $1 \%$ of the world's population and about $20 \%$ of all Arabs) 8 . A previous study in Egypt reported a rate of $1.5 \%$ for social phobia for patients attending an outpatient psychiatric service ${ }^{9}$. To date, there is limited research on the rates of social phobia in university students in the Arab world. A previous study in Egypt reported a rate of $9.3 \%$ for social phobia in a sample of 300 undergraduate university students ${ }^{10}$. In Jordan, a rate of $17.7 \%$ for social phobia was reported in a sample of 729 university students ${ }^{11}$. In Saudi Arabia, Elhadad et al. reported a rate of $59.5 \%$ for social phobia in a sample of 380 medical students ${ }^{12}$.

The aim of this study was to screen for social phobia in a large sample of Egyptian university students from a diverse range of faculties and to investigate the factors (demographic and scholastic) associated with social phobia.

\section{Methods}

\section{Site of the study}

A University-based cross-sectional study which was conducted at Ain Shams University which is located in Greater Cairo and is one of the largest national universities in Egypt. It serves the catchment area of Eastern and Greater Cairo. Data obtained from Central Agency for Public Mobilization and Statistics year book, Egypt, $2016^{13}$ indicated that the number of students enrolled in Ain Shams University different faculties in the year 2016/2017 reached 47.000 students. Ethical approval for the study was granted by the Ain Shams University Faculty of Medicine Ethical Committee.

\section{Participants}

A sample of 2,919 student were randomly allocated from the undergraduates' University files of 9 different faculties: Medicine, Pharmacy, Dentistry, Science, Law, Commerce, Languages, Engineering and Arts. The exclusion criteria were: younger than 18 years of age. Sample Size was calculated using 95\% CI with an accepted margin of error $\pm 6 \%$. The sample was calculated using Epi Info 2002 program.

\section{Tools}

Data was collected by a designed questionnaire which was developed by the researchers and was used to gather data about the students' age, gender, place of birth, place of origin, marital status, employment, number of siblings, BMI, faculty, scholastic year at university, past medical \& psychiatric history, family history, smoking status and use of illicit drugs, followed by assessment using the clinical data sheet of the institute of Psychiatry Ain Shams University (ASUIP) adapting the DSM IV criteria.

Students diagnosed as having Social Phobia were later assessed by using the Brief Social Phobia Scale (BSPS) ${ }^{14}$, which is an observer rated tool for measuring symptoms of social phobia. The scale scored by the observer, has an important role in the evaluation of SAD since patients with SAD tend to devalue their symptoms ${ }^{14}$. A total score of $>20$ indicated symptoms were severe enough to warrant treatment. The BSPS was translated from English to Arabic by two psychiatrists and one psychologist with experience in rating scales. The three versions were compared and a single version was obtained after reaching a consensus. The final Arabic version was back translated by a bilingual senior psychiatrist specialized in anxiety, who did not have access to the original English version, then we compared the back translation to the original.
Using this, subjects were divided into two groups: those with social phobia and those without social phobia

\section{Procedure}

Subjects were recruited during the academic year 2016-2017. All those meeting the inclusion criteria and consenting to participate were included. Purpose of the study was explained to all participants. Demographic, clinical data were collected

\section{Statistical analyses}

Data was recorded and analyzed using the statistical package of social sciences (IBM SPSS 20.0 for windows; SPSS Inc, Chicago, IL, 2010). The results were tabulated, grouped and statistically analyzed using the following tests: Mean, standard deviation (SD) and range for parametric numerical (quantitative) data, or median and range for non-parametric data; frequency and percentage for non-numerical (qualitative) data; Chi-square test for comparing categorical variables or Fisher exact test when the expected frequency was $<5$; independent-sample student t-test to compare means or Mann-Whitney U for non-parametric data; Spearman Correlation Test ( $r$ ) for studying the relationship (direction and power) of quantitative variables. Linear regression was used to test and estimate the dependence of a quantitative variable based on its relationship with a set of independent variables, while logistic regression was used to test the dependence of a qualitative variable based on its relationship with a set of independent variables. A $p$-value of $<0.05$ was considered statistically significant.

\section{Results}

Of the 2,919 subjects who participated in the study, $53.7 \%$ were female and $46.3 \%$ were male with a mean age of 20.33 years $(\mathrm{SD} \pm$ 2.06 , range $=17-28$ years). Out of these, $0.7 \%$ were married $1.4 \%$ had some form of employment, in addition to being students.

Of the 2,919 subjects screened using the BSPS, 1,288 subjects $(44.1 \%)$ reached the threshold for social phobia (score $>20$ ). Table 1 shows socio-demographic and descriptive characteristics of students with and without social phobia. Table 2 shows mean scores (and SD) for whole sample and compares mean scores between subjects with social phobia (BSPS score $>20$ ) and subjects without social phobia for each of the individual items of the sub-domains, total subdomain (fear, avoidance, physiologic) score and total BSPS score. It showed a statistically significant difference between subjects with and without social phobia on all individual items, all the total subdomain scores and the total BSPS score.

\section{Correlation of socio-demographic and descriptive characteristics with BSPS scores}

When we correlated socio-demographic and descriptive characteristics with total scores and scores of the sub-domains of the BSPS (fear, avoidance, physiologic), we found a significant ( $p<$ 0.05 ) positive correlation for age with total avoidance scores, and a significant negative correlation for scholastic year at university with total avoidance, physiologic and total BSPS scores (Table 3).

\section{Risk factors predicting social phobia and total BSPS scores}

Logistic regression analysis was carried out to investigate the predictive factors for-the occurrence of social phobia in our sample. Linear regression analysis was also carried to investigate various factors affecting the total BSPS score. We found that being female and being a student in the Faculty of Medicine were independent risk factors for the development of social phobia and also significantly affected the total BSPS score (Table 4). 
Table 1. Socio-demographic and descriptive characteristics of students with and without social phobia

\begin{tabular}{|c|c|c|c|c|c|}
\hline \multirow[t]{2}{*}{ Mean } & \multicolumn{2}{|c|}{$\begin{array}{l}\text { With social phobia } \\
\qquad(N=1,288)\end{array}$} & \multicolumn{2}{|c|}{$\begin{array}{l}\text { Without social phobia } \\
\qquad(N=1,631)\end{array}$} & \\
\hline & SD & Mean & SD & $p$ & \\
\hline Age & 20.24 & \pm 2.05 & 20.37 & \pm 2.06 & $0.096^{1}$ \\
\hline BMI & 24.24 & \pm 4.08 & 24.27 & \pm 3.75 & 0.8201 \\
\hline Number of siblings & 2.72 & \pm 1.58 & 2.66 & \pm 1.41 & 0.6521 \\
\hline \multicolumn{6}{|l|}{ Gender } \\
\hline Male & & $34.3 \%$ & & $65.7 \%$ & \multirow{2}{*}{$0.0012^{*}$} \\
\hline Female & & $56.6 \%$ & & $43.4 \%$ & \\
\hline \multicolumn{6}{|l|}{ Place of birth } \\
\hline Urban & & $46.8 \%$ & & $53.2 \%$ & \multirow{3}{*}{$0.258^{2}$} \\
\hline Rural & & $51.1 \%$ & & $48.9 \%$ & \\
\hline Outside Egypt & & $51.3 \%$ & & $48.7 \%$ & \\
\hline \multicolumn{6}{|l|}{ Place of origin } \\
\hline Urban & & $46.8 \%$ & & $53.2 \%$ & \multirow{2}{*}{$0.108^{2}$} \\
\hline Rural & & $51.3 \%$ & & $48.7 \%$ & \\
\hline \multicolumn{6}{|l|}{ Marital status } \\
\hline Single & & $46.3 \%$ & & $53.7 \%$ & \multirow{2}{*}{0.5292} \\
\hline Married & & $38.9 \%$ & & $61.1 \%$ & \\
\hline \multicolumn{6}{|l|}{ Employed } \\
\hline No & & $46.3 \%$ & & $53.7 \%$ & \multirow{2}{*}{$0.266^{2}$} \\
\hline Yes & & $37.5 \%$ & & $62.5 \%$ & \\
\hline \multicolumn{6}{|l|}{ Faculty } \\
\hline Medicine & & $46.8 \%$ & & $53.2 \%$ & \multirow{9}{*}{$0.0363^{*}$} \\
\hline Pharmacy & & $45.2 \%$ & & $54.8 \%$ & \\
\hline Dentistry & & $53.3 \%$ & & $46.7 \%$ & \\
\hline Science & & $75.0 \%$ & & $25.0 \%$ & \\
\hline Law & & $30.3 \%$ & & $69.7 \%$ & \\
\hline Commerce & & $32.6 \%$ & & $67.4 \%$ & \\
\hline Languages & & $40.0 \%$ & & $60.0 \%$ & \\
\hline Engineering & & $39.2 \%$ & & $60.8 \%$ & \\
\hline Arts & & $43.3 \%$ & & $56.7 \%$ & \\
\hline \multicolumn{6}{|c|}{ Past medical \& psychiatric history } \\
\hline Yes & & $51.6 \%$ & & $48.4 \%$ & \multirow{2}{*}{$0.0212^{*}$} \\
\hline No & & $45.2 \%$ & & $54.8 \%$ & \\
\hline \multicolumn{6}{|l|}{ Family history } \\
\hline Yes & & $54.5 \%$ & & $45.5 \%$ & \multirow{2}{*}{$0.0222^{*}$} \\
\hline No & & $47.7 \%$ & & $52.3 \%$ & \\
\hline \multicolumn{6}{|l|}{ Smoking } \\
\hline Yes & & $47.3 \%$ & & $52.7 \%$ & \multirow{2}{*}{0.5902} \\
\hline No & & $45.9 \%$ & & $54.1 \%$ & \\
\hline \multicolumn{6}{|l|}{ Use of illicit drugs } \\
\hline Yes & & $30.0 \%$ & & $70.0 \%$ & \multirow{2}{*}{0.1472} \\
\hline No & & $46.2 \%$ & & $53.8 \%$ & \\
\hline
\end{tabular}

BMI: body mass index; SD: standard deviation; p. $p$-value. ${ }^{*}$ Statistically significant.1 Student $t$ test. ${ }^{2}$ Chi-Square tests. ${ }^{3}$ Fisher exact test.

\section{Predictors of high fear, avoidance and physiologic scores on BSPS}

Linear regression analysis was carried to investigate which factors affected fear, avoidance and physiologic scores on the BSPS in our sample. We found that BMI significantly affected physiologic scores, being female significantly affected fear, avoidance and physiologic scores, being a student in the Faculty of Medicine significantly affected avoidance and physiologic scores and number of years at university significantly affected fear scores (Table 5).

\section{Discussion}

The mental health of undergraduate university students is an area of interest worldwide because this is considered a sensitive period in the person's life; which is important for the development of systems and interventions that can reduce or prevent mental health problems ${ }^{15}$. There is a growing body of literature revealing that social anxiety disorder is highly prevalent among adolescents. Yet, most available studies were conducted in Western countries. The current study investigated 2,919 undergraduate university students representing 9 different faculties using the BSPS and found that a total of $44.1 \%$ reached the threshold for social phobia. Compared to those without social phobia, there was a statistically significant difference regarding gender, past medical, past psychiatric and family history.

Our results showed that $44.1 \%$ of subjects reached the threshold for social phobia. This is in line with a previous study done in $\mathrm{Al}$ Azhar University students in Egypt where prevalence of social phobia symptoms reached as high as $68.2 \%$ among GHQ positive subjects ${ }^{16}$. Moreover Ragahb et al. ${ }^{17}$ found that the prevalence of social phobia among Egyptian college students was $30.9 \%$. This is also in line with other Egyptian studies ${ }^{18}$, where a $45.5 \%$ prevalence of social phobia was found in one study of Egyptian college students, and a prevalence of $78 \%$ for generalized social phobia was found in another study ${ }^{19}$. This points out the magnitude of the problem especially in a country like Egypt, where high rates of Social Phobia might be attributed to the socioeconomic situation that prolongs the duration of dependence on family and where attempts at independent living are met with major challenges, foremost economic ones ${ }^{16}$.

While in other Middle Eastern countries a rate of $17.7 \%$ for social phobia was reported in a sample of 729 university students in Jordan ${ }^{11}$, with a rate of $59.5 \%$ reported for social phobia in 380 medical students in Saudi Arabia ${ }^{12}$ and a rate of 9.6\% was found in 1.003 university students in Turkey ${ }^{20}$. Uzonwanne, using the Social Phobia Inventory (SPIN) surveyed 400 students from 5 universities in south west Nigeria and found that $20 \%$ of the students manifested severe social phobia and $16.8 \%$ manifested extremely severe social phobia 7 . In a sample of 380 university students in India, they found social phobia in $19.5 \%$ of subjects ${ }^{21}$. In Western countries, the rate of social phobia among Swedish university students was estimated at $16.1 \%^{22}$, while in first year university students in Australia, the rate was $18.3 \%{ }^{23}$. In Italy, a study done by Dell'Osso et al. on 717 university students using the Social Anxiety Spectrum Self-Report questionnaire, found that $28.6 \%$ reached the threshold for high scorers $^{24}$. Similarly, in the general population, the rate of social phobia varies across different cultures with Asian cultures showing the lowest rate $^{3}$, with $0.2-0.6 \%$ in Korea ${ }^{25}, 0.2 \%$ in China ${ }^{26}$.

Our study showed significantly higher rates of social phobia compared to many other studies. This might reflect the genuine underestimated magnitude of the problem amongst Egyptian university students, given our large sample size. This also might be the difficulty in separating social phobia from poor social skills or shyness developmentally, some studies have shown large ranges in prevalence with prevalence of social phobia depending heavily on where the diagnostic threshold is set 27,28 .

Another important factor to consider is the impact of the culture especially as the defining feature of social phobia is fear of negative evaluation by others which is directly linked to social standards and role expectations, which are culture dependent. According to the "syndromal sensitivity" model, certain traits or behaviors may be more or less prevalent across cultures depending on how consistent they are with cultural ideals or standards and are facilitated and strengthened by reward and praise, whereas traits culturally inconsistent with these cultural ideals are sanctioned or discouraged ${ }^{29}$.

Also, $51.3 \%$ of our sample was originally from rural areas. This might have had an impact on the development of social phobia, which may stem from the social change associated with urbanization and adapting to new social norms. 
Table 2. Brief Social Phobia Scale (BSPS) scores for all sample subjects and comparison of subjects with and without social phobia

\begin{tabular}{|c|c|c|c|c|c|c|c|}
\hline \multirow[b]{2}{*}{ Items of BSPS } & \multicolumn{2}{|c|}{$\begin{array}{l}\text { All subjects } \\
(\mathrm{N}=2,919)\end{array}$} & \multicolumn{2}{|c|}{$\begin{array}{l}\text { With social phobia } \\
\qquad(N=1,288)\end{array}$} & \multicolumn{2}{|c|}{$\begin{array}{l}\text { Without social phobia } \\
\qquad(N=1,631)\end{array}$} & \multirow[t]{2}{*}{$p$-value } \\
\hline & Mean & SD & Mean & SD & Mean & SD & \\
\hline \multicolumn{8}{|l|}{ Fear } \\
\hline Fear of speaking in public or in front of other & 0.64 & \pm 0.92 & 1.03 & 1.07 & 0.30 & 0.58 & $0.0001^{*}$ \\
\hline Fear of talking to people in authority & 0.98 & \pm 1.09 & 1.37 & 1.23 & 0.62 & 0.78 & $0.0001^{*}$ \\
\hline Fear of talking to strangers & 0.65 & \pm 0.97 & 1.02 & 1.12 & 0.33 & 0.64 & $0.0001^{*}$ \\
\hline Fear of being embarrassed or humiliated & 1.30 & \pm 1.37 & 1.86 & 1.50 & 0.79 & 1.02 & $0.0001^{*}$ \\
\hline Fear of being criticized & 0.89 & \pm 1.14 & 1.34 & 1.30 & 0.49 & 0.79 & $0.0001^{*}$ \\
\hline Fear of social gatherings & 0.52 & \pm 0.88 & 0.84 & 1.04 & 0.23 & 0.55 & $0.0001^{*}$ \\
\hline Fear of doing something while being watched & 0.73 & \pm 1.01 & 1.12 & 1.17 & 0.38 & 0.67 & $0.0001^{*}$ \\
\hline Total Fear score & 8.04 & \pm 5.03 & 11.82 & 4.22 & 4.69 & 2.82 & $0.0001^{*}$ \\
\hline \multicolumn{8}{|l|}{ Avoidance } \\
\hline Avoidance of speaking in public or in front of other & 0.82 & \pm 1.05 & 1.45 & 1.13 & 0.36 & 0.68 & $0.0001^{*}$ \\
\hline Avoidance of talking to people in authority & 1.05 & \pm 1.13 & 1.73 & 1.15 & 0.53 & 0.79 & $0.0001^{*}$ \\
\hline Avoidance of talking to strangers & 0.89 & \pm 1.11 & 1.52 & 1.20 & 0.42 & 0.75 & $0.0001^{*}$ \\
\hline Avoidance of being embarrassed or humiliated & 1.67 & \pm 1.48 & 2.38 & 1.39 & 1.14 & 1.33 & $0.0001^{*}$ \\
\hline Avoidance of being criticized & 1.09 & \pm 1.26 & 1.74 & 1.31 & 0.58 & 0.94 & $0.0001^{*}$ \\
\hline Avoidance of social gatherings & 0.63 & \pm 0.98 & 1.19 & 1.15 & 0.22 & 0.55 & $0.0001^{*}$ \\
\hline Avoidance of doing something while being watched & 0.91 & \pm 1.12 & 1.59 & 1.22 & 0.40 & 0.72 & $0.0001^{*}$ \\
\hline Total Avoidance score & 8.62 & \pm 5.30 & 12.65 & 4.16 & 5.16 & 3.38 & $0.0001^{*}$ \\
\hline \multicolumn{8}{|l|}{ Physiologic } \\
\hline Blushing & 1.03 & \pm 1.07 & 1.55 & 1.08 & 0.59 & 0.83 & $0.0001^{*}$ \\
\hline Palpitations & 1.34 & \pm 1.12 & 1.91 & 1.11 & 0.85 & 0.87 & $0.0001^{*}$ \\
\hline Trembling & 0.81 & \pm 0.99 & 1.29 & 1.08 & 0.40 & 0.69 & $0.0001^{*}$ \\
\hline Sweating & 0.67 & \pm 0.96 & 1.03 & 1.11 & 0.35 & 0.66 & $0.0001^{*}$ \\
\hline Total Physiologic score & 3.94 & \pm 3.07 & 5.76 & 3.05 & 2.40 & 2.07 & $0.0001^{*}$ \\
\hline Total BSPS score & 20.54 & \pm 11.22 & 30.23 & 8.12 & 12.25 & 5.29 & $0.0001^{*}$ \\
\hline
\end{tabular}

SD: standard deviation. ${ }^{1}$ Student t test. ${ }^{*}$ Statistically significant.

Table 3. Correlation of socio-demographic and descriptive characteristics with domains of Social Phobia Questionnaire

\begin{tabular}{|l|l|c|c|c|c|}
\hline & & Total Fear Score & Total Avoidance Score & Total Physiologic Score & Total BSPS Score \\
\hline \multirow{2}{*}{ Age } & $r$ & 0.002 & -0.039 & -0.024 & -0.021 \\
\cline { 2 - 6 } & $p$ & 0.901 & $0.038^{*}$ & 0.197 & 0.262 \\
\hline \multirow{2}{*}{ Scholastic year at university } & $r_{s}$ & -0.005 & -0.076 & -0.067 & -0.059 \\
\cline { 2 - 6 } & $p$ & 0.801 & $<0.0001^{*}$ & $<0.0001^{*}$ & $0.002^{*}$ \\
\hline \multirow{2}{*}{ BMI } & $r$ & -0.028 & -0.015 & -0.015 & -0.021 \\
\cline { 2 - 6 } & $p$ & 0.172 & 0.463 & 0.453 & 0.310 \\
\hline \multirow{2}{*}{ Number of siblings } & $r$ & $<0.0001$ & -0.010 & 0.001 & -0.008 \\
\cline { 2 - 6 } & $P$ & 0.983 & 0.630 & 0.964 & 0.724 \\
\hline
\end{tabular}

BMI: body mass index; r: Pearson correlation coefficient, $r$ : Spearman's rho coefficient; $p$ : $p$-value. ${ }^{*}$ Statistically significant.

Table 4. Logistic regression analysis showing factors predicting social phobia and linear regression analysis showing factors affecting total Brief Social Phobia Scale (BSPS) score

\begin{tabular}{|c|c|c|c|c|c|c|c|c|}
\hline & \multicolumn{4}{|c|}{ Logistic Regression } & \multicolumn{4}{|c|}{ Linear Regression } \\
\hline & \multirow[t]{2}{*}{ B } & \multirow[t]{2}{*}{$p$} & \multicolumn{2}{|c|}{$95 \% \mathrm{Cl}$} & \multirow[t]{2}{*}{$B$} & \multirow[t]{2}{*}{$p$} & \multicolumn{2}{|c|}{$95 \% \mathrm{Cl}$} \\
\hline & & & Lower & Upper & & & Lower & Upper \\
\hline Age & 1.019 & 0.886 & 1.171 & 0.796 & 0.252 & -0.505 & 1.010 & 0.513 \\
\hline Female & 2.976 & 2.318 & 3.821 & $0.0001^{*}$ & 6.678 & 5.322 & 8.034 & $0.0001^{*}$ \\
\hline BMl & 1.029 & 0.998 & 1.062 & 0.068 & 0.114 & -0.053 & 0.282 & 0.181 \\
\hline Faculty of medicine & 1.516 & 1.121 & 2.049 & $0.007^{*}$ & -0.375 & -0.697 & -0.052 & $0.023^{*}$ \\
\hline Scholastic year at university & 0.907 & 0.772 & 1.065 & 0.234 & -0.702 & -1.585 & 0.181 & 0.119 \\
\hline Past medical \& psychiatric history & 0.858 & 0.610 & 1.209 & 0.382 & -0.113 & -1.988 & 1.761 & 0.906 \\
\hline Family history & 0.824 & 0.623 & 1.091 & 0.176 & 0.367 & -1.162 & 1.896 & 0.638 \\
\hline
\end{tabular}

B: regression coefficient; BMI: body mass index; p. p-value; $95 \% \mathrm{Cl}$ : 95\% confidence interval. ${ }^{*}$ Statistically significant. 
Table 5. Linear regression analysis showing factors affecting fear, avoidance and physiological scores on Brief Social Phobia Scale (BSPS)

\begin{tabular}{|c|c|c|c|c|c|c|c|c|c|c|c|c|}
\hline & \multicolumn{4}{|c|}{ Fear } & \multicolumn{4}{|c|}{ Avoidance } & \multicolumn{4}{|c|}{ Physiologic } \\
\hline & \multirow[t]{2}{*}{ B } & \multirow[t]{2}{*}{$p$} & \multicolumn{2}{|c|}{$95 \% \mathrm{Cl}$} & \multirow[t]{2}{*}{ B } & \multirow[t]{2}{*}{$p$} & \multicolumn{2}{|c|}{$95 \% \mathrm{Cl}$} & \multirow[t]{2}{*}{ B } & \multirow[t]{2}{*}{$p$} & \multicolumn{2}{|c|}{$95 \% \mathrm{Cl}$} \\
\hline & & & Lower & Upper & & & Lower & Upper & & & Lower & Upper \\
\hline Age & -0.09 & 0.592 & -0.419 & 0.239 & 0.126 & 0.469 & -0.215 & 0.466 & 0.102 & 0.319 & -0.099 & 0.303 \\
\hline Female & 2.42 & $<0.0001^{*}$ & 1.835 & 3.008 & 3.124 & $<0.0001^{*}$ & 2.516 & 3.733 & 1.183 & $<0.0001^{*}$ & 0.824 & 1.542 \\
\hline BMI & 0.02 & 0.509 & -0.048 & 0.097 & 0.043 & 0.263 & -0.033 & 0.119 & 0.048 & $0.034^{*}$ & 0.004 & 0.093 \\
\hline Faculty of medicine & 0.08 & 0.669 & -0.297 & 0.463 & -0.401 & $0.045^{*}$ & -0.794 & -0.009 & -0.266 & $0.024^{*}$ & -0.497 & -0.035 \\
\hline Scholastic year at university & 1.03 & $0.005^{*}$ & 0.315 & 1.746 & 0.489 & 0.197 & -0.254 & 1.232 & 0.467 & 0.034 & 0.035 & 0.899 \\
\hline Past medical \& psychiatric history & -0.305 & 0.465 & -1.123 & 0.513 & -0.022 & 0.959 & -0.868 & 0.824 & 0.339 & 0.180 & -0.157 & 0.835 \\
\hline Family history & 0.012 & 0.972 & -0.656 & 0.680 & 0.202 & 0.567 & -0.490 & 0.894 & 0.051 & 0.804 & -0.353 & 0.455 \\
\hline
\end{tabular}

B: regression coefficient; BMl: body mass index; p: p-value; $95 \% \mathrm{Cl}$ : $95 \%$ confidence interval. ${ }^{*}$ Statistically significant.

Another contributing factor to the prevalence of social phobia in students might be the role of perfectionism. This might be explained by the fact that students experience pressure on many life issues, especially on personal choices such as college studies, where they may be required to comply with pressure from families and feel a need to become perfect in their eyes. It has been shown that negative perfectionism (fear of failure and self-criticism) in students was associated with high anxiety scores and it has also been shown that socially prescribed perfectionism among students is associated with low self-efficacy and ineffective resource management and decrease odds of seeking help ${ }^{30}$.

\section{Social phobia and gender}

Our study showed that being female was a significant predictive factor for the occurrence of social phobia and for fear, avoidance, physiologic and total BSPS scores. That is consistent with the study by Turk et al. where women reported significantly greater fear than men while talking to authority, acting/performing/giving a talk in front of an audience, working while being observed, entering a room when others are already seated, being the center of attention, speaking up at a meeting, expressing disagreement or disapproval to people they do not know very well, giving a report to a group, and giving a party ${ }^{31}$.

As far as Arab countries are concerned, a previous study done in Kuwait investigating the gender difference in anxiety among volunteer undergraduates recruited from 10 Arab countries, showed that females had higher mean anxiety scores than their male counterparts ${ }^{32}$. This can also perhaps be explained in the context of traditional gender-role expectations where, as far as Arab countries are concerned, it has been hypothesized that both child rearing practices and orthodox traditions both have an impact, where shyness is considered a virtue, and where it has been shown that there is high correlation between shyness and social phobia, so that they may not be separate phenomenon but rather an overlapping conditions $\mathbf{s}^{29,31,32}$. Some have also argued that this difference could be due to the collectivistic and orthodox nature of Arab culture where there is respect for authority figures, a fear from criticism and judgment in general and where men generally have more power and authority so that it might increase fear and anxiety in women, especially in social situations ${ }^{33}$.

Contrary to our findings Shah and Kataria found no gender difference for social phobia ${ }^{21}$. Interestingly, a study from Pakistan aimed to explore the prevalence and trend of social anxiety in 1601 female university students from 32 colleges and universities who were observing the veil and found a very low and almost non-significant level of social anxiety in female students who wore the veil ${ }^{33}$.

\section{Age and scholastic year}

A significant negative correlation was found between age and avoidance scores and between scholastic year at university and fear, avoidance and physiologic scores on the BSPS. Younger people perhaps feel more anxious amongst older peers in social situations and use avoidance more frequently as reinforcement in the face of social anxiety especially amongst students, where social phobia is associated with more avoidant behavior in anticipation of a public speaking situation ${ }^{22}$. As regards scholastic years, Inam et al. found higher rates of social phobia (73\%) in second year students compared to an overall rate of $60 \%$ for the whole college in 189 medical students in a Pakistani medical college. This can perhaps be explained by the shift from the traditional spoon-feeding teaching methods that students experience in high school and the eventual decline in social phobia occurs as students get used to the different teaching methods at university ${ }^{34}$.

\section{Medical school and social phobia}

In our study, being a medical student was a significant predictive factor for the occurrence of social phobia and for total, avoidance and physiologic scores on the BSPS. The high prevalence (46.8\%) of social phobia in medical students in our sample was comparable to the study by Elhadad et al. ${ }^{12}$ in Saudi Arabia that showed a 59.5\% prevalence of social phobia in a sample of 380 medical students and where high social phobia scores were associated with poorer academic performance, more likelihood of avoiding oral presentations, more likelihood of performing poorly in clinical examinations and more likelihood of having depressive symptoms ${ }^{12}$.

The link between being a medical student and high levels of social anxiety might be explained by perceived stress. Medical students face unique academic challenges that render them more vulnerable to stress and anxiety than students of other disciplines which probably stems from the environment of medical school itself where there is a high pressure, authoritarian and rigid system that encourages competition rather than cooperation between learners.

Our study was contrary to the study by Shah and Kataria ${ }^{21}$ where social phobia was reported maximally in the faculties of Commerce, Arts and Science, while it was reported to be least in the faculties of Medicine and Engineering. In addition, the Australian study among undergraduate university students reported higher rates of social phobia in students studying for degrees in Arts (38\%) and Health Sciences (36\%), while the lowest rates were seen in Medicine (16\%)23.

\section{Body mass index (BMI)}

Our study showed BMI was a significant predictor of physiological scores on the BSPS. This might stem from the so-called social appearance anxiety which is defined as "the fear that one will be negatively evaluated because of one's appearance" 35 .

\section{Conclusions and limitations}

Our study demonstrated a number of findings in a large sample of university students namely the high rate of social phobia. It also demonstrated a number of significant associated factors 
such as gender, BMI, scholastic year and faculty type with total and subdomain scores on the BSPS. Our study had a number of limitations. The participants were recruited from one university within Cairo (Ain Shams University), so these findings may not be generalizable to other universities within Egypt. Furthermore, we did not have a control arm of age-matched subjects not attending university, so we cannot confirm whether these findings are exclusive to university students or whether they can be extended to others in this age group.

\section{References}

1. Veale D. Treatment of social phobia. Adv Psychiatr Treat. 2003;9(4): 258-64.

2. Stein MB, Stein DJ. Social anxiety disorder. Lancet. 2008;371(9618):1115-25.

3. Hofmann SG, Asnaani A, Hinton DE. Cultural aspects in social anxiety and social anxiety disorder. Depress Anxiety. 2010;27(12):1117-27.

4. Kessler RC, Stein MB, Berglund P. Social phobia subtypes in the National Comorbidity Survey. Am J Psychiatry. 1998;155(5):613-9.

5. Timothy JB, Atezaz S. Social anxiety disorder: a common unrecognized mental disorder. Am Fam Physician. 1999;60(8):2311-9.

6. Russell G, Shaw S. A study to investigate the prevalence of social anxiety in a sample of higher education students in the United Kingdom. J Ment Health. 2009;18(3):198-206.

7. Uzonwanne FC. Prevalence of social phobia, gender and school type among young adults in Nigerian universities. Int J Soc Sci Humanit Res. 2014;2(8):36-45.

8. Population of the World. Population of Egypt (2017). Available from: http:// www.livepopulation.com/country/egypt.html. Accessed on: April 18, 2017

9. Amr M, El-Wasify M, El-Gilany AH, Rees S. Gender differences among patients with social phobia in Egypt. Arab J Psychiatr. 2013;24:52-9.

10. Afifi DY, Ghoz EH, Abdou AA. Social Anxiety among Egyptian University Students [MSc Thesis]. Cairo: Faculty of Medicine, Cairo University; 2012.

11. Momani F, Jaradat AM. Social phobia among university students: prevalence and socio- demographic factors. Jordan Journal of Social Sciences. 2011;4(1):71-88.

12. Elhadad AA, Alzaala MA, Alghamdi RS, Asiri SA, Algarni AA, Elthabet MM. Social phobia among Saudi medical students. Middle East Curr Psychiatry. 2017;24(2):68-71.

13. Central Agency for Public Mobilization and Statistics year book, Egypt, 2016.

14. Davidson JR, Miner CM, De Veaugh-Geiss J, Tupler LA, Colket JT, Potts NL. The brief social phobia scale: a psychometric evaluation. Psychol Med. 1997;27(1):161-6.

15. Bayram N, Bilgel N. The prevalence and socio-demographic correlations of depression, anxiety and stress among a group of university students. Soc Psychiatry Psychiatr Epidemiol. 2008;43(8):667-72.

16. Ragheb K, Taha H. Prevalence of Social Anxiety Symptoms Among a Sample of Egyptian Adolescents. Egypt J Psychiatry. 2009;29(1):5-13.

17. Ragheb K, Abd El-Azem S, Abdel-Wahab M, Attia H, Ismail R, Said M, et al. Social anxiety disorder in children. Curr Psychiatry. 2005;14(3):13-22.
18. Haggag WL, El Sheikh E. Social phobia in college students in Egypt: frequency, correlates and determinants. Curr Psychiatry. 2003;10(1).

19. Bassiony M, Abdel Aal I, Amin H, Saad A, Al Nagar K, Rashed N, et al. Generalized social phobia: prevalence and clinical correlates. Curr Psychiatry. 2001;8(3).

20. Izgiç F, Akyüz G, Doğan O, Kuğu N. Social phobia among university students and its relation to self-esteem and body image. Can J Psychiatry. 2004;49(9):630-4.

21. Shah P, Kataria L. Social phobia and its impact in Indian university students. Internet J Ment Health. 2009;6(2).

22. Tillfors M, Furmark T. Social phobia in Swedish university students: prevalence, subgroups and avoidant behavior. Soc Psychiatry Psychiatr Epidemiol. 2007;42(1):79-86.

23. Wilson I. Screening for social anxiety disorder in first year university students - a pilot study. Aust Fam Physician. 2005;34(11):983-4.

24. Dell'Osso L, Abelli M, Pini S, Carlini M, Carpita B, Macchi E, et al. Dimensional assessment of DSM-5 social anxiety symptoms among university students and its relationship with functional impairment. Neuropsychiatr Dis Treat. 2014;10:1325-32.

25. Cho MJ, Kim JK, Jeon HJ, Suh T, Chung IW, Hong JP, et al. Lifetime and 12-month prevalence of DSM-IV psychiatric disorders among Korean adults. J Nerv Ment Dis. 2007;195(3):203-10.

26. Shen YC, Zhang MY, Huang YQ, He YL, Liu ZR, Cheng H, Tet al. Twelve-month prevalence, severity, and unmet need for treatment of mental disorders in metropolitan China. Psychol Med. 2006;36(2):257-67.

27. Furmark T, Tillfors M, Everz PO, Marteinsdottir I, Gefvert O, Fredrikson M. Social phobia in the general population: prevalence and sociodemographic profile. Soc Psychiatry Psychiatr Epidemiol. 1999;34(8):416-24.

28. Stein MB, Walker JR, Forde DR. Setting diagnostic thresholds for social phobia: considerations from a community survey of social anxiety. Am J Psychiatry. 1994;151(3):408-12.

29. Iancu I, Bodner E, Joubran S, Zion IB, Ram E. Why not the best? Social anxiety symptoms and perfectionism among Israeli Jews and Arabs: a comparative Study. Compr Psychiatry. 2015;59:33-44.

30. Afshar H, Roohafza H, Sadeghi M, Saadaty A, Salehi M, Motamedi $\mathrm{M}$, et al. Positive and negative perfectionism and their relationship with anxiety and depression in Iranian school students. J Res Med Sci. 2011;16(1):79-86.

31. Turk CL, Heimberg RG, Orsillo SM, Holt CS, Gitow A, Street LL, et al. An investigation of gender differences in social phobia. J Anxiety Disord. 1998;12(3):209-23.

32. Alansari BM. Gender differences in anxiety among undergraduates from sixteen Islamic countries. Soc Behav Personality. 2006;34(6):651-60.

33. Hussain N. Prevalence of Social Anxiety in Female Students Who Veil in Universities: Evidence from Pakistan. Presentation to the 9th International Academic Conference, Florence, Italy; 2015.

34. Inam SN, Saqib A, Alam E. Prevalence of anxiety and depression among medical students of private university. J Pak Med Assoc. 2003;53(2):44-7.

35. Hart TA, Flora DB, Palyo SA, Fresco DM, Holle C, Heimberg RG. Development and examination of the social appearance anxiety scale. Assessment. 2008;15(1):48-59. 